\title{
Neural Correlates of the Shamanic State of Consciousness
}

\section{OPEN ACCESS}

Edited by:

Gopikrishna Deshpande,

Auburn University, United States

Reviewed by:

Lorenzo Pasquini,

Klinikum rechts der Isar der Technischen Universität München,

Germany

David Luke,

University of Greenwich, United Kingdom

${ }^{*}$ Correspondence:

Richard E. Harris

reharris@med.umich.edu

tThese authors have contributed equally to this work

Specialty section: This article was submitted to Cognitive Neuroscience, a section of the journal Frontiers in Human Neuroscience

Received: 25 September 2020 Accepted: 25 February 2021

Published: 18 March 2021

Citation:

Huels ER, Kim H, Lee U, Bel-Bahar T, Colmenero AV, Nelson A,

Blain-Moraes S, Mashour GA and Harris RE (2021) Neural Correlates of the Shamanic State of Consciousness. Front. Hum. Neurosci. 15:610466. doi: 10.3389/fnhum.2021.610466

\author{
Emma R. Huels ${ }^{1,2,3 \dagger}$, Hyoungkyu Kim ${ }^{2,3 \dagger}$, UnCheol Lee 1,2,3, Tarik Bel-Bahar ${ }^{4}$, Angelo \\ V. Colmenero ${ }^{5}$, Amanda Nelson ${ }^{2}$, Stefanie Blain-Moraes ${ }^{6}$, George A. Mashour ${ }^{1,2,3}$ \\ and Richard E. Harris ${ }^{1,7 *}$
}

\begin{abstract}
'Neuroscience Graduate Program, University of Michigan, Ann Arbor, MI, United States, ${ }^{2}$ Department of Anesthesiology, University of Michigan, Ann Arbor, MI, United States, ${ }^{3}$ Center for Consciousness Science, University of Michigan, Ann Arbor, MI, United States, ${ }^{4}$ Division of Translational Epidemiology, New York State Psychiatric Institute, New York, NY, United States, ${ }^{5}$ The Graduate Center, City University of New York, New York, NY, United States, ${ }^{6}$ School of Physical and Occupational Therapy, McGill University, Montreal, QC, Canada, ${ }^{7}$ Chronic Pain and Fatigue Research Center, Department of Anesthesiology, University of Michigan, Ann Arbor, MI, United States
\end{abstract}

Psychedelics have been recognized as model interventions for studying altered states of consciousness. However, few empirical studies of the shamanic state of consciousness, which is anecdotally similar to the psychedelic state, exist. We investigated the neural correlates of shamanic trance using high-density electroencephalography (EEG) in 24 shamanic practitioners and 24 healthy controls during rest, shamanic drumming, and classical music listening, followed by an assessment of altered states of consciousness. EEG data were used to assess changes in absolute power, connectivity, signal diversity, and criticality, which were correlated with assessment measures. We also compared assessment scores to those of individuals in a previous study under the influence of psychedelics. Shamanic practitioners were significantly different from controls in several domains of altered states of consciousness, with scores comparable to or exceeding that of healthy volunteers under the influence of psychedelics. Practitioners also displayed increased gamma power during drumming that positively correlated with elementary visual alterations. Furthermore, shamanic practitioners had decreased low alpha and increased low beta connectivity during drumming and classical music and decreased neural signal diversity in the gamma band during drumming that inversely correlated with insightfulness. Finally, criticality in practitioners was increased during drumming in the low and high beta and gamma bands, with increases in the low beta band correlating with complex imagery and elementary visual alterations. These findings suggest that psychedelic drug-induced and non-pharmacologic alterations in consciousness have overlapping phenomenal traits but are distinct states of consciousness, as reflected by the unique brain-related changes during shamanic trance compared to previous literature investigating the psychedelic state.

Keywords: shamanism, consciousness, EEG, altered states of consciousness, Trance 


\section{INTRODUCTION}

Recent studies regarding the neuroscience of consciousness have leveraged altered states of consciousness as model systems, such as the psychedelic state (Johnson et al., 2019). However, the use of pharmacologic agents to induce altered states of consciousness introduces potential confounds by pharmacologic manipulation of "off-target" brain receptors. For example, while studies have reported the 5HT2A receptor as crucial for the induction of altered states of consciousness via serotonergic psychedelics (Kraehenmann et al., 2017; Preller et al., 2018), these compounds may also bind to other serotonergic and non-serotonergic receptor sites (Halberstadt and Geyer, 2011), or possibly $5 \mathrm{HT} 2 \mathrm{~A}$ receptor sites not crucial to the psychedelic experience. For example, 5HT2A receptors are present in the gut (Fiorica-Howells et al., 2002; O'Mahony et al., 2015) and it is reasonable to question the importance of such receptors in the induction of the psychedelic state. In contrast, shamanic trance, which is an altered state of consciousness anecdotally similar to the psychedelic state, can be achieved with or without the use of psychedelic substances by listening to repetitive drumming (Krippner, 2000). During shamanic trance, the shaman (in cases of indigenous tribes) or shamanic practitioner (in cases of neo-shamanism in the western world) enters an altered state of consciousness, termed the shamanic state of consciousness (Harner, 1980), to offer physical, psychological, or spiritual healing for others. In this state, shamanic practitioners endure changes in consciousness similar to individuals under the influence of psychedelic substances, such as mystical experiences, feelings of disembodiment or flight, or feelings of ego dissolution (Winkelman, 2013). Despite its universality in ancient civilizations (Tributsch, 2018), increasing popularity in the present day, and semblance to psychedelically-induced altered states, empirical studies of shamanic trance remain limited. Elucidating the neural correlates of this unique altered state of consciousness may offer valuable insights into the neurobiology of consciousness, particularly how changes in the phenomenal content of consciousness arise in the absence of pharmacologic intervention.

To date, only two neuroimaging studies have investigated brain activity in shamanic practitioners during shamanic trance. Using functional MRI, Hove et al. found increased hub activity in key regions in default mode and controlrelated networks during the shamanic state (Hove et al., 2016). Functional connectivity between these regions was also increased during trance, suggesting an interaction between these networks. Research using electroencephalographic activity during the shamanic state of consciousness revealed increased low $(13-20 \mathrm{~Hz})$ and high $(21-50 \mathrm{~Hz})$ beta activity, as well as an anterior-posterior shift in power during the transition from normal to trance state (Flor-Henry et al., 2017). Although these studies contributed to our knowledge of the shamanic state of consciousness, the Hove et al. (2016) trial lacked non-shamanic practitioner controls and Flor-Henry et al. (2017) examined data from only a single shamanic practitioner. Furthermore, these past studies did not correlate neurobiological metrics with the subjective experiences of this state. These gaps must be addressed to compare this unique state of consciousness to other altered states.

Thus, we investigated the shamanic state of consciousness using a validated assessment of altered states of consciousness and high-density electroencephalography (EEG) in both experienced shamanic practitioners and naive control participants. While spectral analyses remain standard in many EEG studies, recent investigations regarding altered states of consciousness have utilized other computational measures to characterize brain activity, such as functional connectivity (Lee et al., 2017; Li et al., 2019), signal diversity (Li and Mashour, 2019; Timmermann et al., 2019; Pal et al., 2020), and criticality (Kitzbichler et al., 2009; Kim et al., 2016, 2017; Lee et al., 2019). Each of these measures informs on various attributes of the neural signal-functional relationships, complexity (i.e., number of different patterns in the signal), and the balance between order and disorder, respectively - that better elucidate the neural correlates underlying brain states. Thus, we characterized EEG changes during shamanic drumming using spectral analysis, connectivity, signal diversity, and criticality and determined their relationship to changes in a variety of domains used to characterize altered states of consciousness. Furthermore, we compared these self-reported changes in consciousness during shamanic trance to the altered state of consciousness induced by psychedelic substances.

\section{MATERIALS AND METHODS}

\section{Ethics}

This study was approved by the University of Michigan Institutional Review Board (HUM00089064). Written informed consent was obtained from all participants before study procedures.

\section{Participants}

Two separate populations were recruited for this study: 24 experienced shamanic healing practitioners and 24 ageand sex-matched control participants. Shamanic practitioner inclusion criteria were as follows: five or more years of practice in shamanic techniques; performed a minimum of 40 shamanic healing sessions over the last 5 years, primarily focused on healing individual people; received training in at least one shamanic tradition or style under expert supervision; used rhythmic drumming during shamanic journeying and healing practice; and possessed the ability to enter a shamanic trance in $15 \mathrm{~min}$ and complete a shamanic healing session in $30 \mathrm{~min}$ while sitting still and quiet. Practitioners were excluded if they had a history of traumatic brain injury or epilepsy. Control participants were age- and sex-matched to shamanic practitioners with the following inclusion criteria: no current or past experience or interest in shamanic practice or media involving shamanism; no current or past history of meditation, spiritual healing, or spiritual training; no current or past history of hallucinogen, entheogen, or psychedelic drug use; no current or past experience with trance or hypnosis, or interest relating to media of such themes; and no current or past history of being a drummer, frequent listening to drumming music, or a 
history of professional musicianship. Control participants were excluded if they had a current or past diagnosis of depression, anxiety, schizophrenia, or other serious psychological disorders, untreated major physical or psychological illness, a history of traumatic brain injury, or a history of epilepsy. Subjects were excluded from analyses for technical difficulties related to EEG acquisition $(n=5)$, EEG data quality issues $(n=4)$, or lack of questionnaire data $(n=2)$, resulting in 18 shamanic practitioners and 19 control participants in the final cohort for analysis. Demographics for these participants are illustrated in Supplementary Table 1.

\section{Pre-study Procedures}

Before their study visit, shamanic practitioners were asked to practice entering a shamanic trance during the $25 \mathrm{~min}$ of rhythmic drumming music to be played during the experiment (Harner, 1997) while control participants were asked to listen to the same pre-recorded drumming without any attempts at entering a shamanic trance. This recording, entitled " 30 -min solo drumming journey with callback" from Michael Harner's Shamanic Journey Solo and Double Drumming, consisted of rhythmic solo drumming without the accompaniment of other instruments. We combined the original 15-min drumming piece (Harner, 1997) with the middle $10 \mathrm{~min}$ of this piece repeated, for a total of $25 \mathrm{~min}$.

\section{Study Design}

Participants underwent baseline EEG recordings with their eyes open for $5 \mathrm{mins}$ and closed for 5 mins. Following baseline recordings, each participant underwent three experimental blocks: cognitive testing, shamanic drumming, and classical music listening. The order of blocks was counterbalanced between groups. Participants were asked to rest again with their eyes opened and closed for $5 \mathrm{~min}$ following the last experiment. EEG data were collected continuously throughout all experiments. Example study day timelines are illustrated in Figure 1A. Given that this manuscript is centered on brain activity during the shamanic state of consciousness, cognitive testing results will not be discussed.

\section{Shamanic Healing Experiment}

During the shamanic healing experiment, shamanic practitioners and controls listened to the same pre-recorded drumming (Harner, 1997) they listened to at home before the study visit. Shamanic practitioners were asked to conduct shamanic healing during this time, during which they entered an altered state of consciousness to glean information to be used for the physical, psychological, or spiritual healing of a client not present during the study. Control participants were instructed to rest quietly and both groups were asked to keep their eyes closed during the drumming period. Following the drumming period, shamanic practitioners and controls completed an Altered States of Consciousness questionnaire known as the OAV scale (Bodmer et al., 1994), which contains 66 items about their subjective experience. Of note, we used the English version of this questionnaire, which has been previously validated in German (Bodmer et al., 1994).

\section{Classical Music Experiment}

The classical music experiment was meant to function as a negative control for the shamanic drumming experiment and entailed $15 \mathrm{~min}$ of classic music listening, which consisted of two songs played in succession. The first song, which was Beethoven's "Sonata No.5 in F Major for Violin and Piano, Op. 24, "Spring": I. Allegro," was a mixture of piano and violin (Beethoven, 1801/2013, track 20). The second piece, Mozart's "Sonata for Piano No. 15 in F Major, K. 533: I. Allegro," was piano only (Mozart, 1788/2009, track 43). These pieces were chosen because they had some degree of repetition, similar to the shamanic drumming recording. Additionally, unlike most musical options, classical music is free of language or singing, furthering its similarities to the shamanic drumming recording. Both shamanic practitioners and control participants were asked to listen with their eyes closed during this time and shamanic practitioners were explicitly asked to not enter a shamanic trance. Participants again filled out the OAV scale following classical music listening.

\section{Qualitative Analysis}

The 66-item OAV Scale questionnaire data were pooled into 11 domains according to previous literature (Studerus et al., 2010) including experience of unity, spiritual experience, blissful state, insightfulness, disembodiment, impaired control and cognition, anxiety, complex imagery, elementary visual alterations, audio-visual synesthesia, and changed meaning of percepts. The percent of the theoretical scale maximum was calculated for each domain for each individual, followed by the mean and standard error of the mean for each group (shamanic practitioners or control participants).

\section{EEG Acquisition}

Participants were fitted with a 129-channel HydroCel Geodesic Sensor Net (Electrical Geodesics, Inc./Magstim EGI Eugene, OR, USA) according to head circumference. EEG data were acquired at a $500-1,000 \mathrm{~Hz}$ sampling rate with a vertex reference using NetAmps 400 amplifier and Netstation 4.5 software. Channel impedances were reduced to below $50 \mathrm{k} \Omega$ per the manufacturer's instruction.

\section{EEG Analysis}

Using the MATLAB open-source toolbox EEGLab (Delorme and Makeig, 2004), data were visually inspected for artifact and preprocessed via resampling to $500 \mathrm{~Hz}$ as necessary, bandpass filtering between $1-45 \mathrm{~Hz}$, and re-referencing to the global average. Data were epoched into 10-s non-overlapping windows; all analyses were conducted on sequential 10-s windows. Subsequent analyses focused on absolute power, functional connectivity, neural signal diversity, and criticality (Figure 1B) within each of the following frequency bands: delta 1-4 Hz, theta 4-8 Hz, low alpha $8-10 \mathrm{~Hz}$, high alpha 10-13 Hz, low beta $13-20 \mathrm{~Hz}$, high beta $20-30 \mathrm{~Hz}$, and gamma $30-45 \mathrm{~Hz}$ (except for connectivity, which was limited to $30-35 \mathrm{~Hz}$ to ensure the reliability of this measure). While we did not collect electromyography data to control for 
A

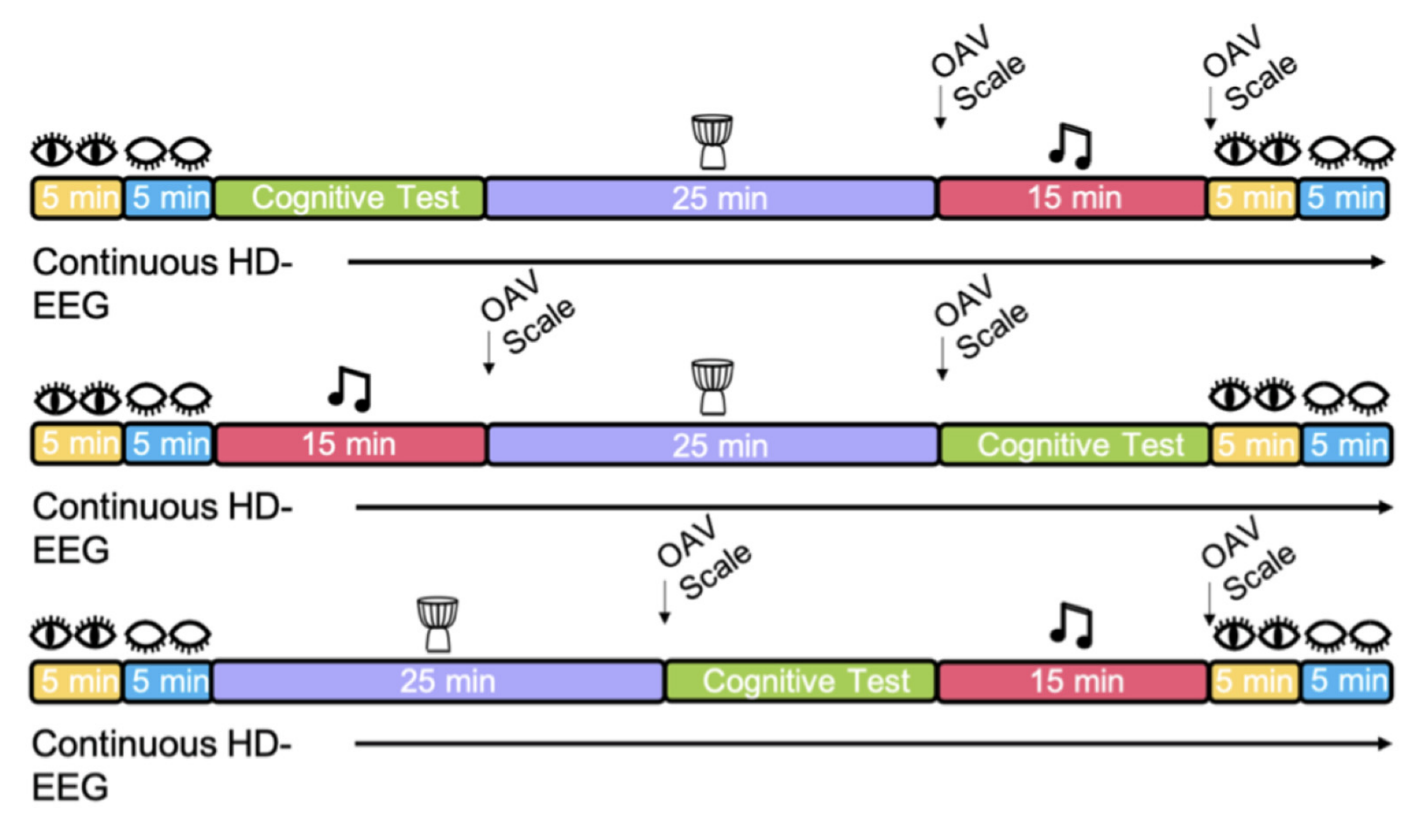

B
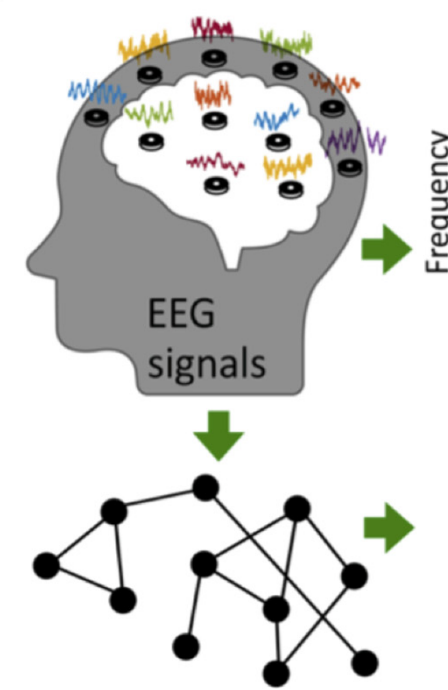

Functional network

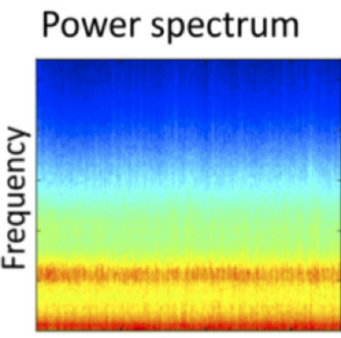

Time
Lempel-Ziv complexity

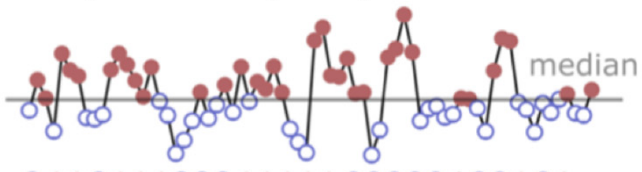

$01101110001111100000100101 \ldots$

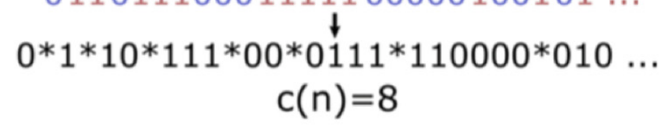

Functional connectivity

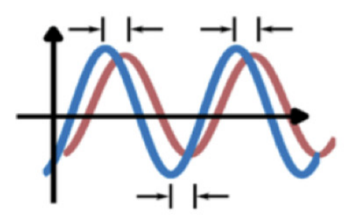

weighted Phase lag Index : phase lead-lag relationship

\section{Criticality}

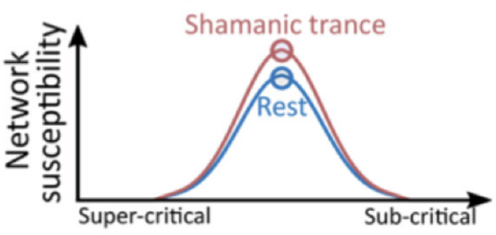

Pair Correlation Function

: variation of synchronization

FIGURE 1 | Study design. (A) Example timelines of a typical study day based on a given experiment block. Each individual was randomized to the order they underwent cognitive testing, shamanic drumming, and classical music listening. Eyes open and eyes closed resting periods occurred at the beginning and end of the experimental day. The Altered States of Consciousness Questionnaire, known as the OAV scale, was administered following classical music and shamanic drumming. (B) All participants underwent high-density electroencephalogram (EEG) recordings during drumming, classical music, and periods of eye-opening and closure. Following preprocessing, EEG signals were used to compute subsequent measures within each frequency band, including the power spectrum, functional connectivity measured by weighted Phase Lag Index, signal diversity measured by Lempel-Ziv complexity (image referenced from Leemburg and Bassetti, 2018), and criticality measured by the pair correlation function.

muscle artifact, we did not anticipate differences in muscle contamination across participants and conditions, meaning any differences in the gamma frequency band were presumably from electroencephalographic differences. EEG measures were computed for the full duration of periods of eye closure (5 min) and shamanic drumming $(25 \mathrm{~min})$. Analyses of brain activity during classical music were performed on the first song period ( $10 \mathrm{~min})$.

\section{Spectral Analysis}

Spectral power was calculated using the "spectrogram.m" function in the MATLAB Signal Processing Toolbox (time 
window: 3 s hamming window, overlap: 50\%) based on the short-time Fourier transform. For each state (eyes closed, classical music, drumming), absolute power within each frequency band was averaged across all time windows and channels.

\section{Functional Connectivity}

Functional connectivity was assessed using the average weighted Phase Lag Index (wPLI), which measures the phase lead-lag relationship between two signals. If two signals are found to be functionally coupled within a given frequency band, the resulting wPLI value will be high within that band. wPLI is robust to the EEG volume conduction problem (Stam et al., 2007; Vinck et al., 2011) and improves Phase Lag Index with weights of the imaginary part of the cross-spectrum, reducing noise and volume conduction effects. When the imaginary part of the crossspectrum is $\mathfrak{\Im}[X]$,

$$
{ } \mathrm{PPLI}=\frac{|E\{\Im\{X\}\}|}{E\{|\Im\{X\}|\}}=\frac{|E\{|\Im\{X\}| \operatorname{sgn}(\Im\{X\})\}|}{E\{|\Im\{X\}|\}}
$$

where the signed Phase Lag Index is the numerator normalized by the denominator, the imaginary part of the cross-spectrum. The wPLI was calculated for each frequency band and averaged across time and all channels in each state.

\section{Neural Signal Diversity}

Recent studies examining psychedelic-induced altered states of consciousness have sought to characterize changes in signal diversity (Schartner et al., 2017; Timmermann et al., 2019), which reflects the number of unique (i.e., diverse) patterns within a signal. Given the aforementioned overlap between the psychedelic state and the shamanic state of consciousness, we measured and compared the neural signal diversity of each state using Lempel-Ziv complexity (LZc), which calculates the temporal complexity of the signal by computing the number of distinct subsequences through the whole signal (Lempel and Ziv, 1976; Figure 1B, image referenced from Leemburg and Bassetti, 2018). The time series of each EEG epoch was binarized using the median value $(\mathrm{M})$ of each time series (Zhang et al., 2001; Leemburg and Bassetti, 2018) as a threshold.

$$
s(n)=\{0 \text { if } x(n)<M, 1 \text { if } x(n) \geq M
$$

where $x(n)$ is each data point of the original time series and the median value is $M$. The median value of each epoch is used for the robustness to outliers and the invariance from the signal amplitude.

A new subsequence of consecutive numbers ("words") was counted as $c(n)$ through the binarized time series and normalized by the theoretical maximum number of words $b(n)=n \log _{2} n$.

$$
C(n)=c(n) b(n)
$$

LZc was computed for each frequency band in each state and averaged across time and channels. To better compare changes in $\mathrm{LZc}$ during shamanic trance to those in previous literature (i.e., psychedelic states), we computed LZc of the broadband (1-45 Hz) signal, as well as within individual frequency bands.

\section{Criticality}

Criticality, a balanced state between order and disorder (Bak et al., 1988), is observed ubiquitously in physical and biological systems and is accompanied by various functional benefits, including large information storage, optimal information transmission, and flexible response to external stimuli (Muñoz, 2018). Many computational and experimental studies suggest that human and animal brain functioning resides near a critical point during conscious wakefulness (Lee and Mashour, 2018) and a loss of criticality induces altered states of consciousness such as sleep, anesthesia, and unresponsive wakefulness syndrome (Kitzbichler et al., 2009; Kim et al., 2016, 2017; Lee et al., 2019). We assessed criticality using the pair correlation function (PCF), which is the variance of network synchronization, reflecting network susceptibility to internal and external perturbations (e.g., from stimuli; Yoon et al., 2015). PCF is maximal at a critical point and gradually decreases as a system moves toward a sub- or super-critical state (Kim and Lee, 2019). Recently, the reliability of PCF in evaluation of the level of consciousness was supported with a computational model and EEG during general anesthesia (Kim and Lee, 2019).

$$
P C F=N\left\{<\operatorname{Re}^{2}[z(t)]>_{t}-<\operatorname{Re}[z(t)]>_{t}^{2}\right\}
$$

where $\operatorname{Re}[z(t)]$ is the real part of the $z(t)$.

$$
z=r e^{i \psi}=\frac{1}{N} \sum_{j=1}^{N} e^{i \theta_{j}}
$$

where $\Psi$ is the order parameter phase. The absolute value $r=|z|$ represents the degree of synchronization. The $r$ is equal to zero when the phases of nodes are uniformly distributed and one when all the nodes have the same phase.

\section{Statistical Analysis}

For comparison of OAV domain scores, Wilcoxon signed-rank tests were used to assess differences in paired samples, and Wilcoxon rank-sum tests were used to assess differences between shamanic practitioners and controls. With permission from Studerus et al. (2010) and using open-access data, we used the mean and standard deviation to compare OAV domain scores of shamanic practitioners and individuals under the influence of psychedelic substances using unpaired $t$-tests. This comparison included data collected from 43 studies that administered psilocybin (115-350 $\mu \mathrm{g} / \mathrm{kg}$ orally; low to high dose), ketamine (6-12 $\mu \mathrm{g} \mathrm{kg}^{-1} \mathrm{~min}^{-1}$ intravenously; medium to high dose), or MDMA (1.5-1.7 $\mathrm{mg} / \mathrm{kg}$ orally; medium dose) to healthy volunteers. Given the aggregation of studies, it is unclear to what degree these data included naive vs. experienced psychedelic users. A two-way mixed analysis of variance was used to assess differences in EEG measures between groups (shamanic practitioner vs. control; between-subject factor) and across conditions (eyes closed vs. classical music vs. drumming; within-subject factor), as well as any interaction between these two factors (group $\times$ condition). Exploratory post-hoc analysis of group differences within each condition was conducted using unpaired $t$-tests. Given our small sample size and the exploratory nature of post hoc tests, we did not adjust for multiple 
comparisons. Spearman correlations were conducted to assess relationships between EEG measures during drumming that were statistically significantly different between groups and OAV domain scores in shamanic practitioners that were significantly different from the control group during shamanic drumming. Again, we did not adjust for multiple comparisons given the exploratory nature or the limited sample size. Findings were considered statistically significant if $p<0.05$.

\section{Data Availability}

All data and code used for this project will be made available upon request of the authors with a data-sharing agreement between institutions.

\section{RESULTS}

\section{Shamanic Practitioners Enter an Altered State of Consciousness During Drumming} We first evaluated differences in OAV domain scores to verify that shamanic practitioners entered an altered state of consciousness. Shamanic practitioners had significantly greater scores than control participants in 8 of the 11 domains during shamanic healing, including complex imagery $[Z=4.22$, $p<0.001,95 \%$ CI $(0.30,0.57)]$, experience of unity $[Z=4.04$, $p<0.001,95 \%$ CI $(0.33,0.65)]$, spiritual experience $[Z=4.87$, $p<0.001,95 \% \mathrm{CI}(0.33,0.60)]$, blissful state $[Z=4.39, p<0.001$, $95 \%$ CI $(0.30,0.60)]$, disembodiment $[Z=4.4, p<0.001$, $95 \%$ CI $(0.23,0.67)]$, insightfulness $[Z=4.06, p<0.001$, $95 \%$ CI $(0.23,0.57)]$, elementary visual alterations $[Z=2.43$, $p=0.0159,95 \%$ CI $(0.033,0.40)]$, and changed meaning of percepts $[Z=3.22, p=0.00136,95 \% \mathrm{CI}(0.033,0.40$; Figure 2A) $]$. These differences did not exist when comparing OAV domain scores during the classical music experiment (Figure 2B). Furthermore, shamanic practitioners were significantly different in all of the above domains except elementary visual alterations when comparing their scores during shamanic drumming to classical music $(Z=2.98$ to $Z=3.62, p<0.01$ to $p<0.001$ ), indicating a majority of the increased scores were specific to the shamanic drumming experiment. Finally, shamanic practitioners had increased scores in audio-visual synesthesia $[Z=-2.35$, $p=0.0199,95 \%$ CI $(-0.42,-0.033)]$ and control participants had increased scores in blissful state $[Z=-2.42, p=0.017,95 \%$ CI $(-0.23,-0.017)]$ during classical music listening compared to drumming.

\section{Shamanic Practitioners Endure Changes in Consciousness Similar to Yet Distinct From the Psychedelic State}

Given the overlap between the shamanic state of consciousness and the psychedelic experience, we thought it would be informative to compare the OAV domain scores from shamanic practitioners with those of individuals under the influence of psychedelic substances. Studerus et al. pooled OAV scores from healthy individuals under the influence of psilocybin, ketamine, and 3,4-Methyl-enedioxy-methamphetamine (MDMA; Studerus et al., 2010). With author permission, we replotted the mean and standard error of the mean for each drug condition from
Studerus et al. with our data from shamanic practitioners and compared each of the domains for each drug condition to shamanic practitioners using unpaired $t$-tests. As can be seen in Figure 2C, shamanic practitioners experienced several domains at or above the level of individuals under the influence of various psychedelics. Specifically, the domains of complex imagery (ketamine: $p<0.001$; psilocybin: $p=0.0077$; MDMA: $p<0.001$ ), experience of unity (ketamine: $p=0.0052$; psilocybin: $p<0.001$; MDMA: $p<0.001$ ), spiritual experience (ketamine: $p<0.001$; psilocybin: $p<0.001$; MDMA: $p<0.001)$, and insightfulness (ketamine: $p<0.001$; psilocybin: $p<0.001$; MDMA: $p<0.001)$ appear to be greater for shamanic practitioners during trance compared to healthy controls under any of these very potent psychedelic compounds. Shamanic practitioners were similar to MDMA in the domains of blissful state (ketamine: $p<0.001$; psilocybin: $p=0.0036$; MDMA: $p=0.14$ ), audio-visual synesthesia (ketamine: $p=0.031$; psilocybin: $p=0.0017$; MDMA: $p=0.96$ ), and impaired control and cognition (ketamine: $p<0.001$; psilocybin: $p=0.0045$; MDMA: $p=0.16$ ), comparable to ketamine in the domain of disembodiment (ketamine: $p=0.46$; psilocybin: $p<0.001$; MDMA: $p<0.001$ ), and similar to ketamine and psilocybin in elementary visual alterations (ketamine: $p=0.28$; psilocybin: $p=0.27$; MDMA: $p<0.001$ ). Additionally, individuals under the influence of ketamine had significantly higher anxiety scores (ketamine: $p=0.037$; psilocybin: $p=0.27$; MDMA: $p=0.99$ ) compared to shamanic practitioners during drumming. Finally, practitioners were similar to all three drugs in the domain of changed meaning of percepts (ketamine: $p=0.92$; psilocybin: $p=0.52$; MDMA: $p=0.63$ ). Additional statistical output measures are detailed in Supplementary Table 2.

\section{Absolute Gamma Power Increases During Drumming in Shamanic Practitioners and Correlates With Elementary Visual Alterations}

Next, we assessed differences in absolute power between shamanic practitioners and controls during eyes closed, classical music, and drumming (Figure 3A). There was a statistically significant interaction between group and condition on gamma power (Group $\times$ Condition: $F_{(1.7,59.1)}=6.3, p=0.005$ ). Posthoc exploratory $t$-tests revealed shamanic practitioners had significantly greater gamma power during drumming compared to controls $\left[t_{(29)}=2.16, p=0.039,95 \%\right.$ CI $(0.040,1.43)$; Figure 3B]. Furthermore, gamma power during drumming positively correlated with the degree of elementary visual alterations $\left(r_{\mathrm{s}}=0.52, p=0.025\right.$; Figure $\left.3 \mathrm{C}\right)$ in shamanic practitioners.

\section{Shamanic Practitioners Have Altered Functional Connectivity in the Low Alpha and Beta Bands During Drumming}

We also examined differences in functional connectivity within each state in shamanic practitioners and control participants (Figure 4A). There was a significant main effect of group on low alpha $\left(F_{(1,35)}=5.6, p=0.024\right)$ and low beta 


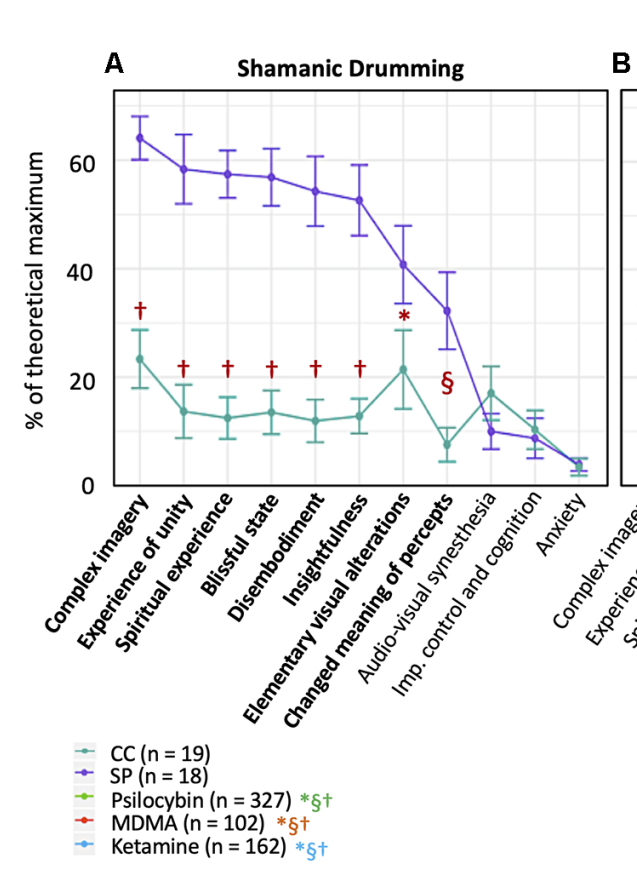

B Classical Music

C

Shamanic Drumming vs.

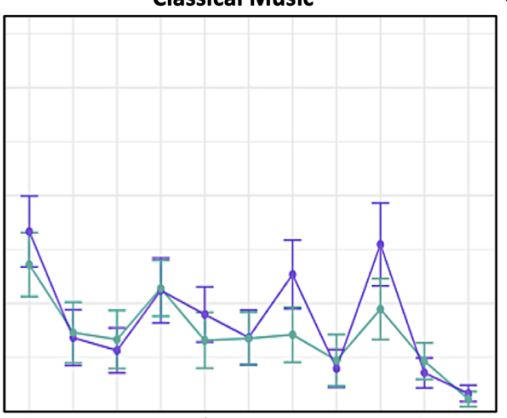

Psychedelic State

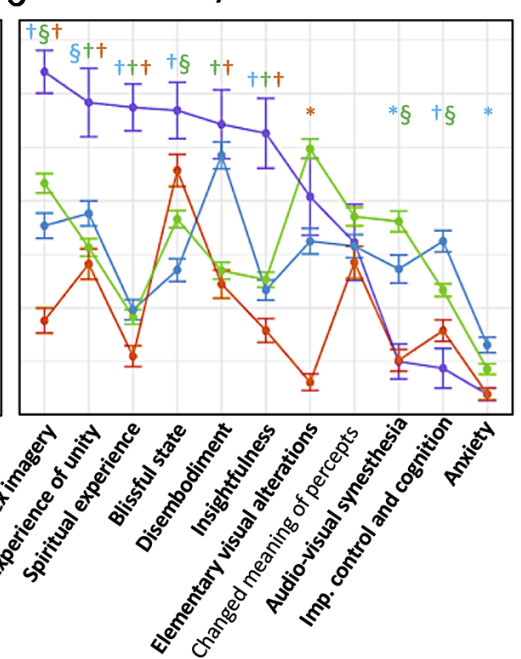

FIGURE 2 | Shamanic practitioners enter an altered state of consciousness during shamanic drumming. (A) Shamanic Practitioners (SP, purple, $n=18$ ) were significantly different from control participants (CC, green, $n=19$ ) during drumming in 8 of the 11 OAV domains. Each point represents the mean of all SP or CC for the percent of the theoretical scale maximum for that domain. Error bars represent the standard error of the mean. (B) SP did not differ from CC during classical music listening on any of the domains. (C) We replotted the OAV mean and standard error for healthy controls taking psilocybin (green, $n=327$ ), ketamine (blue, $n=162$ ), or MDMA (orange, $n=102$ ) from Studerus et al. (2010) and compared these scores to those acquired from SP. Overall, SP had increased scores in several domains compared to all three drug conditions, as well as similar scores in the remaining domains compared to various drug conditions.

$\left(F_{(1,35)}=4.41, p=0.043\right)$ connectivity. Post hoc $t$-tests revealed that shamanic practitioners had significantly decreased low alpha connectivity during drumming $\left[t_{(27.2)}=-2.45\right.$, $p=0.021,95 \%$ CI $(0.00731,0.0828)]$ and music $\left[t_{(27.8)}=-2.22\right.$, $p=0.035,95 \%$ CI $(0.00374,0.0938)]$ compared to control participants (Figure 4B). Additionally, shamanic practitioners had significantly greater connectivity in the low beta band during drumming $\left[t_{(22.1)}=2.11, p=0.047,95 \% \mathrm{CI}(0.000352,0.0432)\right]$ and music $\left[t_{(24.9)}=2.25, p=0.033,95 \% \mathrm{CI}(0.00263,0.0584)\right]$ compared to controls (Figure 4B). These statistically significant measures of connectivity did not correlate with any of the OAV domains.

\section{Lempel-Ziv Complexity in the Gamma Band Decreases During Drumming in Shamanic Practitioners and Correlates With Feelings of Insightfulness}

We evaluated differences in EEG signal diversity using Lempel-Ziv complexity (LZc; Figure 5A). There was a significant main effect of group on $\mathrm{LZc}$ in the gamma band $\left(F_{(1,35)}=4.79\right.$, $p=0.035)$. Post-hoc testing revealed that shamanic practitioners had decreased LZc in the gamma band during drumming $\left(t_{(19.4)}=-2.53, p=0.02,95 \%\right.$ CI $\left.[-0.0274,-0.00262]\right)$ compared to control participants (Figure 5B). Furthermore, LZc in the gamma band was negatively correlated with feelings of insightfulness $\left(r_{s}=0.5, p=0.034\right.$; Figure 5C). While analysis of broadband $\mathrm{LZc}$ revealed a statistically significant interaction between group and condition (Group $\times$ Condition: $F_{(2,70)}=3.7$, $p=0.03$ ), post hoc $t$-tests did not reveal any significant differences between groups.

\section{Shamanic Practitioners Experience Increased Criticality in Beta and Gamma Bands During Drumming That Correlates With OAV Domain Scores}

Finally, we examined differences in criticality using the pair correlation function (PCF; Figure 6A) during eyes closed, music, and drumming. Given that the shamanic state of consciousness is characterized by a high level of mental activity (Figure 2A), we would anticipate increased PCF during shamanic trance which would suggest increased criticality and, in turn, increased metastability (i.e., patterns of global synchronization over time) and network susceptibility to internal and external perturbations. There was a main effect of group on PCF in the low beta $\left(F_{(1,35)}=4.59, p=0.039\right)$ and high beta bands $\left(F_{(1,35)}=5.94\right.$, $p=0.02)$. For PCF in the gamma band, there were main effects of both group $\left(F_{(1,35)}=10.09, p=0.003\right)$ and condition $\left(F_{(2,70)}=5.93, p=0.004\right)$. Exploratory post-hoc $t$-tests revealed that, during drumming, shamanic practitioners had greater PCF in the low beta $\left(t_{(34.5)}=3.32, p=0.00213,95 \% \mathrm{CI}\right.$ $[0.00176,0.00729]$; Figure 6B1), high beta $\left(t_{(33.2)}=3.29\right.$, $p=0.00235,95 \%$ CI $[0.00273,0.0115]$; Figure 6B2), and 

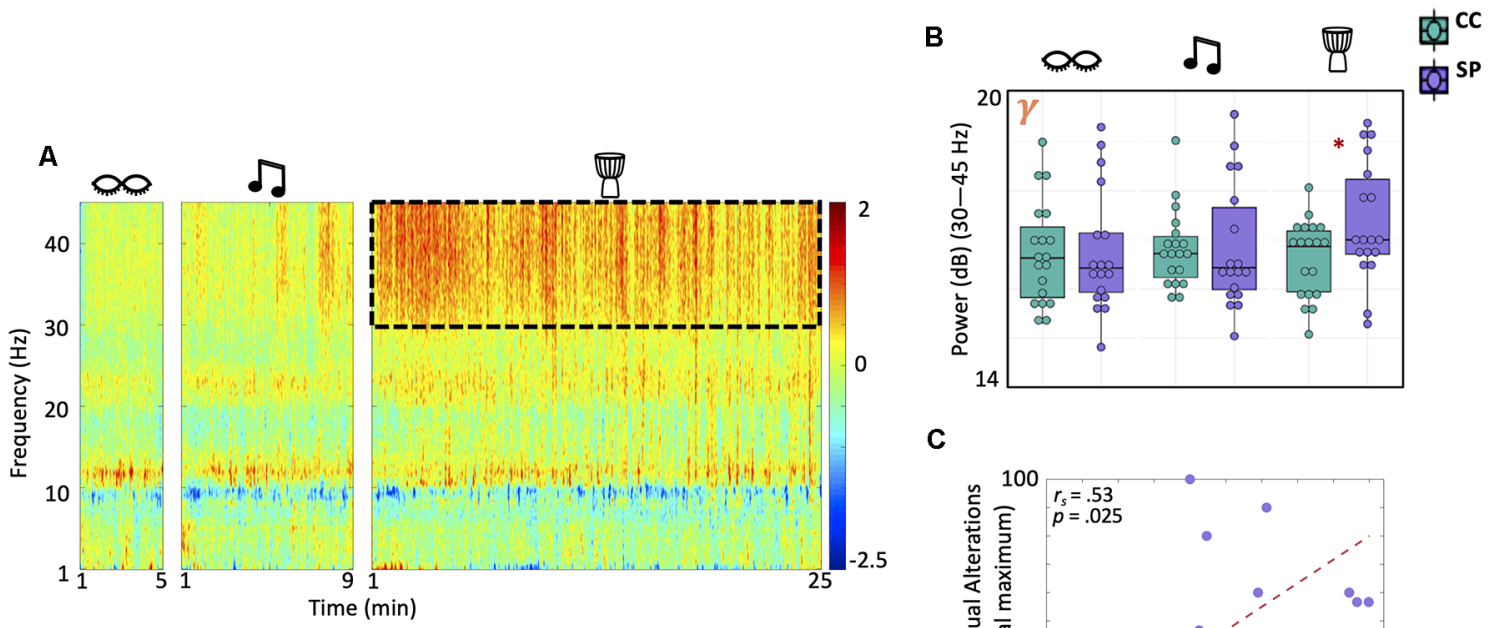

C

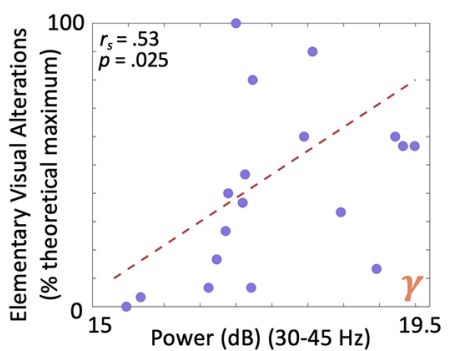

$p<.05: *$

FIGURE 3 | Gamma power is greater in shamanic practitioners during the drumming and correlates with elementary visual alterations. (A) Power spectrogram comparing the difference in power across frequencies (1-45 Hz) between shamanic practitioners (SP; $n=18)$ and control participants (CC; $n=19)$ during eyes closed, classical music, and drumming periods. Warmer colors represent greater power in SP and cooler colors indicate more power in CC. The dotted line represents the gamma frequency band $(30-45 \mathrm{~Hz})$, which was significantly different between groups during shamanic drumming. (B) Box plots representing the mean absolute power of SP (purple) or CC (green) during eyes closed, drumming, and music. Whiskers represent the lower (25\%) and upper (75\%) quartiles and the center band of each box represents the median for the group. SP had significantly greater gamma power during the drumming period compared to CC. Significance is indicated by the key in the lower righthand corner of the figure. (C) We correlated absolute gamma power with each of the eight ASC domain scores that were significantly different between groups during drumming in Figure 2A, revealing a positive correlation between absolute gamma power and the degree of elementary visual alterations.

gamma $\left(t_{(33.7)}=4.1, p<0.001,95 \%\right.$ CI $[0.00754,0.0224]$; Figure 6B3) bands compared to controls. Furthermore, PCF in the low beta band positively correlated with the degree of complex imagery $\left(r_{s}=0.53, p=0.023\right.$; Figure 6C1) and elementary visual alterations $\left(r_{s}=0.56, p=0.015\right.$; Figure 6C2). Shamanic practitioners also had greater PCF in the high beta band during classical music $\left(t_{(35)}=2.1, p=0.043\right.$; Figure 6B2) and the gamma band during classic music $\left(t_{(31.5)}=2.75\right.$, $p=0.00987)$ and eyes closed $\left(t_{(31)}=2.16, p=0.039\right.$; Figure 6B3).

\section{DISCUSSION}

\section{Summary}

In the largest and most comprehensive neuroimaging study of shamanic practitioners to date, we characterized the shamanic state of consciousness using high-density EEG and a well-validated assessment for altered states of consciousness known as the OAV scale. We revealed shamanic practitioners to be significantly different from controls in a majority of the $\mathrm{OAV}$ domains during drumming. Furthermore, these differences were similar or greater in magnitude than those reported by individuals under the influence of ketamine, psilocybin, or MDMA. In addition to the main effects of the group on a variety of EEG measures, post-hoc exploratory analyses revealed that shamanic practitioners had increased absolute gamma power during drumming, which positively correlated with the degree of elementary visual alterations. Additionally, practitioners had decreased low alpha and increased low beta connectivity during drumming and classical music. Furthermore, signal diversity in the gamma band was decreased in shamanic practitioners during drumming, which negatively correlated with feelings of insightfulness. Finally, PCF was increased in the low beta, high beta, and gamma bands during drumming, with increases in the low beta band positively correlating with complex imagery and elementary visual alterations. Additionally, PCF in the high beta band was increased during classical music in shamanic practitioners compared to control participants, as was PCF in the gamma band during eyes closed and classical music. These findings suggest that, during shamanic trance, shamanic practitioners enter an altered state of consciousness that is accompanied by changes in a variety of EEG measures that correlate with self-reported changes in experience. Specifically, differences in gamma power, gamma complexity, and low beta PCF were exclusive to shamanic drumming and correlated with items on the OAV scale, meaning they likely represent changes in brain activity specific to the shamanic state of consciousness. In contrast, EEG changes spreading across drumming and classical music, such as changes in low alpha or low beta functional connectivity or high 


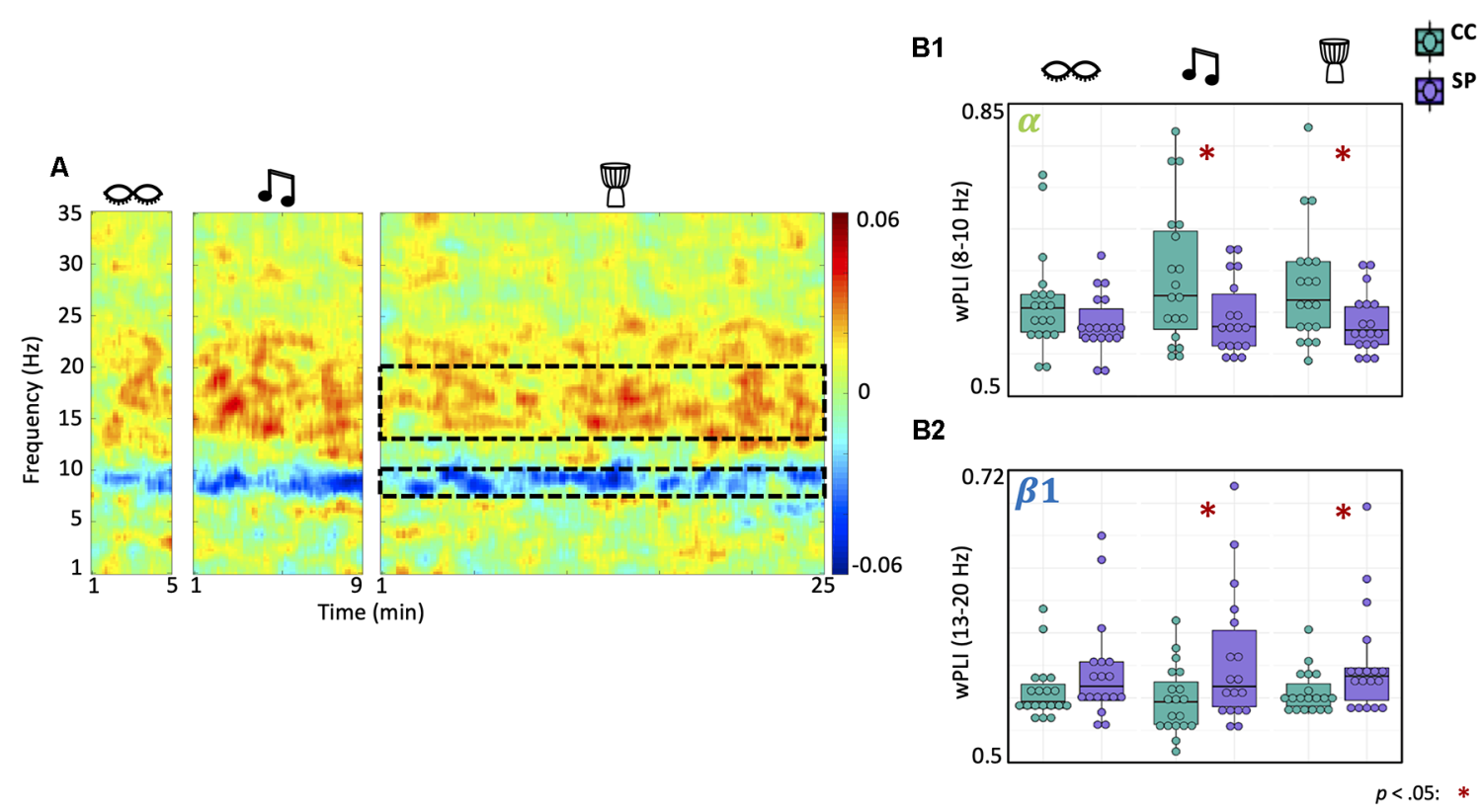

FIGURE 4 | Shamanic practitioners have increased low beta and decreased low alpha connectivity during drumming and classical music. (A) Differences in functional connectivity, measured by weighted Phase Lag Index (wPLI), between shamanic practitioners (SP; $n=18$ ) and control participants (CC; $n=19)$. Warmer colors represent greater connectivity in SP while cooler colors indicate greater connectivity in CC. The dotted lines represent the low alpha (8-10 Hz) and low beta (13-20 Hz) frequency bands, which were significantly different between groups. (B1,B2) Box plots illustrating the average wPLI in the low alpha (B1) or low beta (B2) frequency bands in SP (purple) and CC (green) during eyes closed, drumming, and music. Whiskers represent the lower (25\%) and upper (75\%) quartiles and the center band of each box represents the median for the group. SP experienced statistically significant decreases in wPLI in the low alpha band during classical music and drumming compared to CC (B1), as well as statistically significant increases in the low beta band (B2). Significance is indicated by the key in the lower righthand corner of the figure.

beta or gamma PCF, may represent trait-related changes in shamanic practitioners.

\section{The Shamanic State of Consciousness, Gamma Oscillations, and Other Absorptive States of Consciousness}

To our knowledge, the only previous investigation of shamanic trance in shamanic practitioners using EEG was by Flor-Henry et al., who found increased absolute low (13-20 Hz) and high (21-50 Hz) beta power during shamanic drumming (FlorHenry et al., 2017). These findings are similar to those of the current study, as we found increased gamma power $(30-45 \mathrm{~Hz})$ during drumming in shamanic practitioners compared to control participants. While we did not see differences in the low beta band, the presence of increased gamma power in both investigations supports the involvement of gamma oscillations in the shamanic state of consciousness. Gamma oscillations have been implicated in a variety of cognitive processes, including consciousness (Havlík, 2017), maintenance of visual images within working memory (Ewerdwalbesloh et al., 2016), and visual perception (Martinovic and Busch, 2011), which is relevant given the correlation between gamma power and elementary visual alterations. Furthermore, previous investigations of brain activity during meditation have revealed increased gamma activity (Lutz et al., 2004; Cahn et al., 2010; Fell et al., 2010; Berkovich-Ohana et al., 2012; Braboszcz et al., 2017; Kakumanu et al., 2018), with some attributing elevations in gamma to enhanced perceptual clarity (Cahn et al., 2010), spontaneous visual imagery (Luft et al., 2019), or increased attentional effort (Braboszcz et al., 2017). Other trance states, such as possession trance, are frequently characterized by power changes in the theta, alpha, or beta bands (Oohashi et al., 2002; Bastos et al., 2016; Kawai et al., 2017), without analysis of the gamma band. Thus, while changes in the other frequency bands are absent during shamanic trance, it remains unknown whether our findings of increased gamma power are comparable. While it is difficult to pinpoint the exact reason for increased gamma power during shamanic trance, our findings are in agreement with previous literature regarding the shamanic state of consciousness and suggest similarities between shamanic trance and meditative states.

In addition to increases in gamma power, shamanic practitioners demonstrated decreased neural signal diversity and increased criticality (i.e., PCF) in the gamma band during drumming when compared to control participants. While studies of signal diversity and criticality in non-pharmacologically induced altered states of consciousness remain scarce, a recent EEG study during meditation assessed changes in entropy and metastability (Martínez Vivot et al., 2020), which is directly related to criticality (e.g., increased metastability is associated with increased criticality). Martínez Vivot et al. (2020) revealed meditative practice to be characterized by increased entropy and 

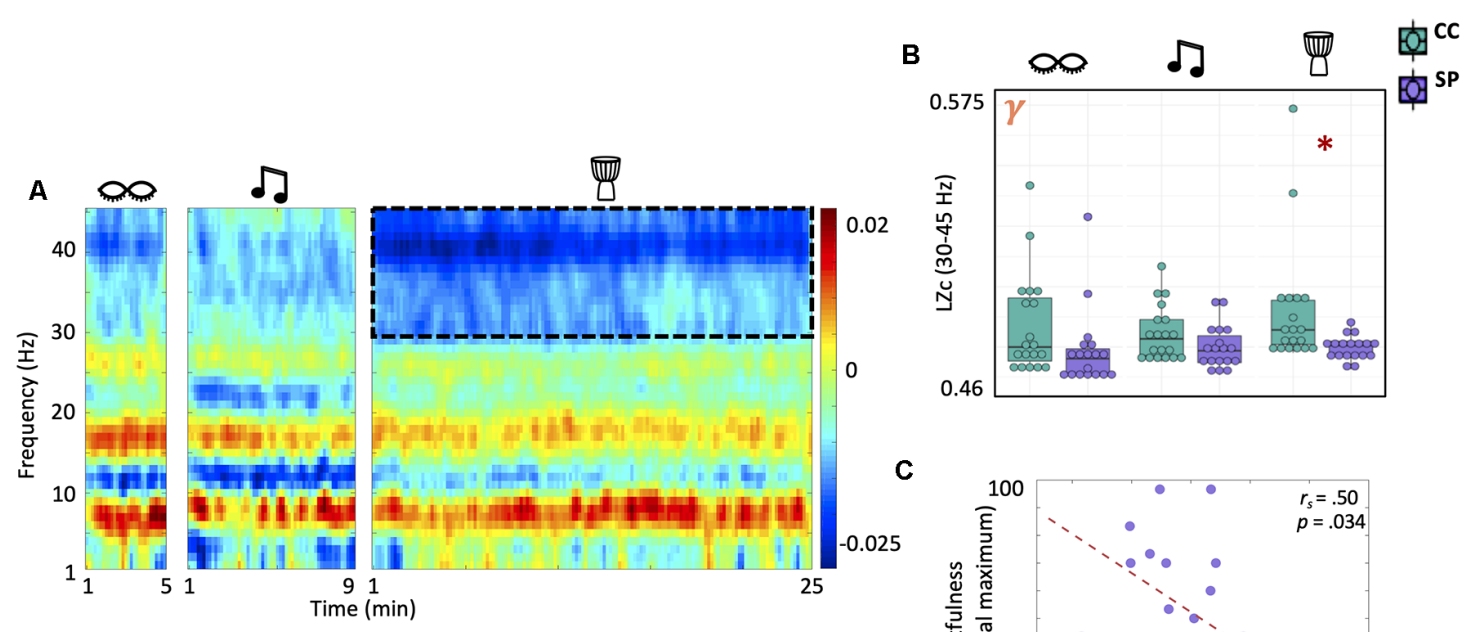

C

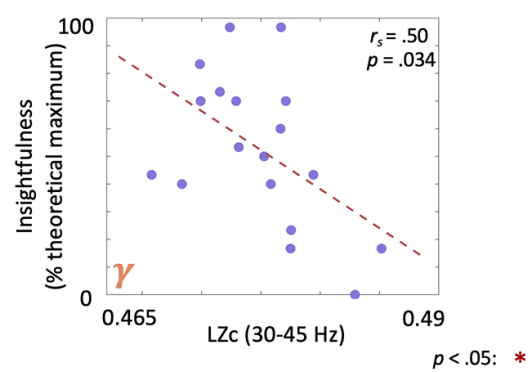

FIGURE 5 | Shamanic practitioners have decreased neural signal diversity in the gamma band during drumming which is negatively correlated with feelings of insightfulness. (A) Comparison of the Lempel-Ziv complexity (LZc), a surrogate measure of neural signal diversity, across frequencies in shamanic practitioners (SP; $n=18$ ) and controls (CC; $n=19$ ). Warmer colors represent greater $L Z c$ in SP while cooler colors indicate greater LZc in CC. The dotted lines represent the gamma band $(30-45 \mathrm{~Hz}$ ), which was significantly different between groups. (B) Box plots comparing the average LZc in the gamma band for SP (purple) and CC (green) during eyes closed, classical music, and drumming. Whiskers represent the lower (25\%) and upper (75\%) quartiles and the center band of each box represents the median for the group. SP experienced statistically significant decreases in LZc in the gamma band compared to CC. Significance is indicated by the key in the lower righthand corner of the figure. (C) Correlations between LZc in the gamma band and ASC domain scores revealed a negative correlation between gamma LZc and feelings of insightfulness.

decreased metastability in the gamma band. This is the inverse of our findings, where shamanic practitioners had decreased neural signal diversity and increased criticality in the gamma band, which, by definition, indicates increased metastability and brain network susceptibility. Given the paucity of research evaluating these measures in the context of shamanic trance or other absorptive states, it is difficult to discern the reason for this difference. One such explanation could be a difference in computational methods, as Martínez Vivot et al. (2020) evaluated changes in sample entropy (compared to Lempel-Ziv complexity) and utilized different techniques for measuring metastability. Additionally, despite the similarities between shamanic trance and deep meditative trance, they are distinct states of consciousness that are achieved through different means and for different purposes, meaning an inverse of findings may not be surprising.

The comparison of EEG changes during the shamanic state of consciousness and other spiritual or religious practices remains less clear. Previous studies have detailed increased alpha power (Abdullah and Omar, 2011; Al-Galal and Alshaikhli, 2017) and changes in relative alpha and theta power (Vaghefi et al., 2015) while listening to the recitation of the Quran, as well as increased approximate and sample entropy (Vaghefi et al., 2019). Additionally, increased absolute alpha power has been noted during catholic prayer (Barcelona et al., 2020), as well as increased relative alpha power during Muslim prayer
(Doufesh et al., 2012, 2014). While these findings related to Abrahamic religious practices differ from those of the current study, an investigation of EEG changes in Carmelite nuns during the recall of mystical experiences found changes in theta and gamma power (Beauregard and Paquette, 2008), with the authors attributing increases in gamma to individuals entering a more absorptive state. Thus, the shamanic state of consciousness appears to overlap more with religious or spiritual practices coupled with mystical experiences characterized by perturbations in conscious experience (i.e., unity with God, visual imagery) rather than solely religious actions. Future investigations of mystical experiences during religious or spiritual practice, as well as other absorptive or contemplative states, are needed.

\section{Shamanic Practitioners Enter an Altered State of Consciousness Similar Yet Distinct From the Psychedelic State}

Our analyses revealed significant differences between shamanic practitioners and control participants in 8 of the $11 \mathrm{OAV}$ domains during drumming, with elevations in those 8 domains mirroring or exceeding domains altered during the psychedelic state. This suggests that shamanic practitioners are entering an altered state of consciousness during trance and confirms the anecdotal overlap with the psychedelic state. However, 

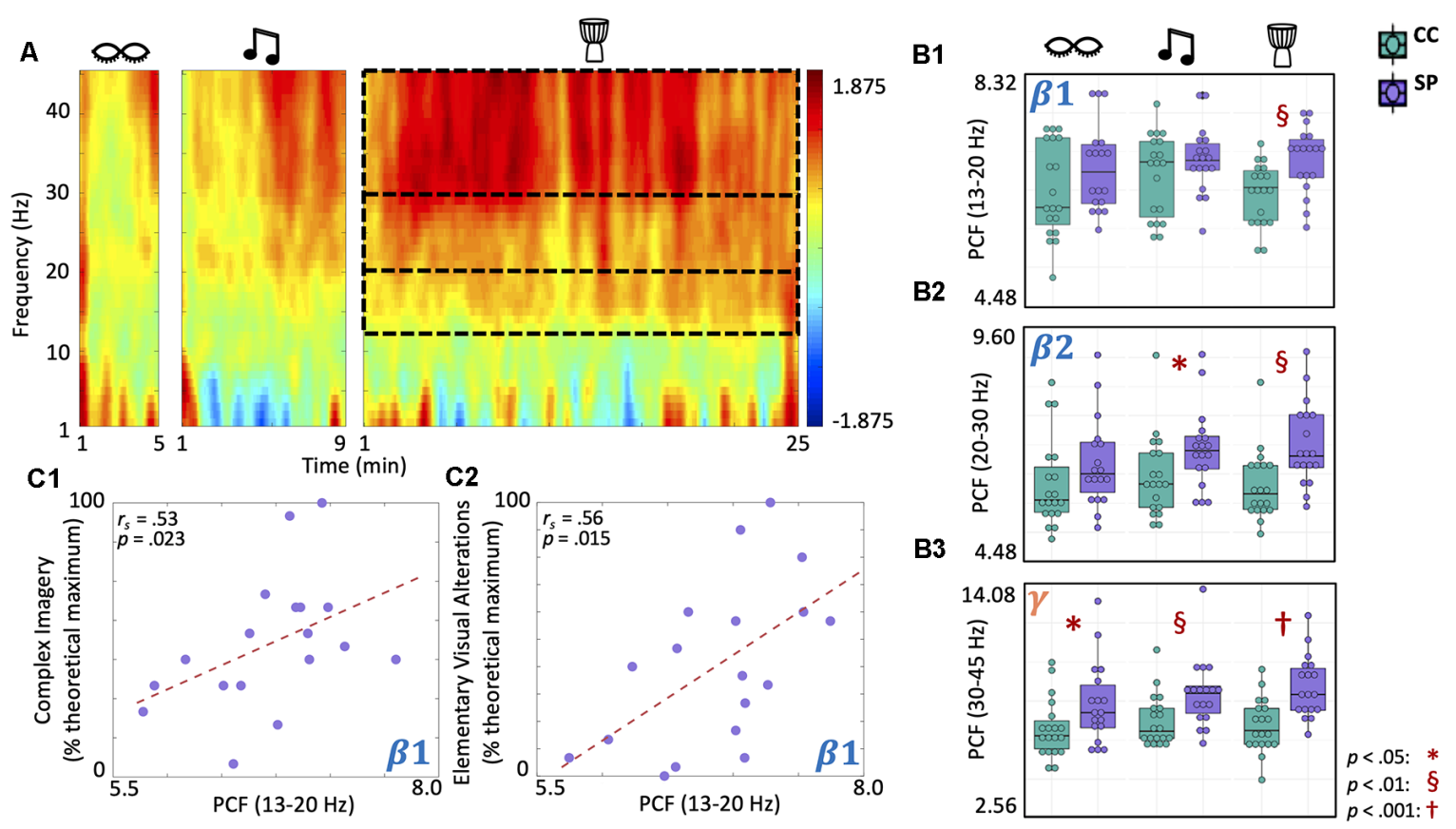

FIGURE 6 | Shamanic practitioners have increased criticality in beta and gamma bands during drumming, with low beta increases correlating with complex imagery and elementary visual alterations. (A) Comparison of criticality, measured using a pair correlation function (PCF), across different frequencies between shamanic practitioners (SP; $n=18$ ) and control participants (CC; $n=19)$. Warmer colors indicate greater PCF in SP while cooler colors represent increased PCF in CC. Dotted lines represent the low (13-20 Hz) and high (20-30 Hz) beta and gamma (30-45 Hz) bands, which were significantly different between SP and controls. (B1-B3) Box plots illustrating the average PCF value for the low (B1) and high (B2) beta and gamma (B3) bands in SP (purple) and CC (green) during eyes closed, classical music, and drumming. Whiskers represent the lower (25\%) and upper (75\%) quartiles and the center band of each box represents the median for the group. SP had greater PCF in the low and high beta (B1,B2) and gamma (B3) bands during drumming compared to CC, with increases extending to the classic music condition in high beta (B2) and gamma (B3) bands and the eyes closed condition in the gamma band (B3). Significance is indicated by the key in the lower righthand corner. (C1,C2) Spearman correlations revealed a positive correlation between PCF in the low beta band and both complex imagery (C1) and elementary visual alterations (C2).

the shamanic state of consciousness was different from the drug response in multiple OAV domains, suggesting that shamanic trance is a distinct altered state and does not perfectly correlate with the drug experience of any of the three substances examined by Studerus et al. (2010), even ones used for shamanic practice (e.g., psilocybin). This assertion is supported by the lack of congruency between previous EEG and magnetoencephalographic (MEG) findings during the psychedelic state and the results of the current study. Past studies have demonstrated widespread decreases in broadband power (Riba et al., 2002, 2004; Muthukumaraswamy et al., 2013; Kometer et al., 2015; Carhart-Harris et al., 2016; Pallavicini et al., 2019), with an emphasis on decreases in the alpha band that correlate with the degree of subjective effects (Kometer et al., 2013; Carhart-Harris et al., 2016; Valle et al., 2016; Timmermann et al., 2019). Given our results demonstrating an overlap between self-reported changes in consciousness during shamanic trance and the psychedelic state, it is surprising that shamanic practitioners not only lacked changes in alpha power but experienced increases in power isolated to the gamma band that correlated with the degree of elementary visual alterations. Although increased gamma power has been reported during select studies of Ayahuasca exposure (Don et al., 1998; Schenberg et al., 2015)—an Amazonian brew commonly used for shamanic practice that contains the psychoactive substance N,N-dimethyltryptamine-it was found to be unrelated to psychometric measures (Schenberg et al., 2015). Thus, while the shamanic state of consciousness involves similar changes in phenomenology as the psychedelic state, the underlying neurobiology of shamanic trance appears to be distinct from altered states of consciousness induced by psychedelics.

While literature investigating functional connectivity during the psychedelic state using EEG and MEG remains scarce, one study did find changes in the lagged phase synchronization in the delta band during psilocybin to be associated with feelings of insightfulness and spiritual experience (Kometer et al., 2015). Our findings suggest that while shamanic practitioners differed from controls in low alpha and low beta connectivity, these changes are not associated with the degree of assessed altered states of consciousness. Furthermore, changes in connectivity also occurred during classical music and, according to Figure 4A, to some degree at baseline, possibly reflecting long-term (i.e., trait) changes in low alpha and low beta connectivity in shamanic practitioners.

Previous literature examining changes in neural signal diversity during the psychedelic state have consistently revealed increases in LZc (Schartner et al., 2017; Timmermann et al., 2019) 
that correlate with the strength of psychedelic-induced changes in phenomenal content (Schartner et al., 2017; Timmermann et al., 2019), particularly in visual domains. Yet, we found that the shamanic state of consciousness was characterized by decreases in neural signal diversity in the gamma band that negatively correlated with feelings of insightfulness, suggesting that decreased diversity in the neural signal was indicative of a more profound experience. One possible explanation for this discrepancy is that previous literature has focused on broadband signal diversity rather than diversity within a given frequency band. However, analysis of broadband LZc did not reveal any significant differences between groups. When coupled with the lack of decreased alpha power, our findings suggest that while shamanic trance may be anecdotally similar to the psychedelic state, they are discrete altered states of consciousness, most likely with different underlying mechanisms by which changes in the subjective experience originate. This is further supported by previous literature demonstrating increased activity in the default-mode network (DMN) during shamanic trance (Hove et al., 2016) rather than the characteristic decreases found during the psychedelic state (Carhart-Harris et al., 2012, 2016; Palhano-Fontes et al., 2015), with decreases in alpha power that correlated with feelings of ego dissolution localizing to nodes of the DMN (Muthukumaraswamy et al., 2013). Future studies should aim to investigate shamanic trance with and without the use of pharmacological intervention to further characterize the relationship between shamanic and psychedelic states.

\section{The Shamanic State of Consciousness and the Entropic Brain Hypothesis}

According to the entropic brain hypothesis, consciousness arises within a critical zone rather than a critical point (Carhart-Harris et al., 2014), which is supported by a series of studies demonstrating that a hierarchical modular structure of the brain network extends a critical point to a critical region (Moretti and Muñoz, 2013). The extended critical region allows for increased sustainability, which in turn enables the brain to maintain criticality under perturbations. Furthermore, the entropic brain hypothesis suggests that normal waking consciousness exists within a band positioned in the middle of this critical zone and changes in consciousness occur when pushed beyond the lower or upper limits of this band. For example, sedatives and anesthetics shift the brain downward, closer to a lower limit (i.e., sub-critical or extremely ordered), and diminish conscious contents. On the contrary, psychedelics shift the brain upward, closer to the upper limit of the critical zone (i.e., super-critical or extremely disordered) with richer conscious contents (CarhartHarris et al., 2014). This was recently demonstrated by Atasoy et al., who provided empirical evidence for heightened criticality during Lysergic acid diethylamide (LSD) using human fMRI blood oxygen level-dependent (BOLD) signals (Atasoy et al., 2017).

In the current study, we found that shamanic trance increased PCF in beta and gamma bands. As mentioned previously, increased PCF is indicative of increased criticality, and in turn, increased brain network susceptibility to perturbations and metastability. Based on the assumption that the brain during normal waking consciousness resides near criticality, increased PCF of shamanic practitioners compared to the control group implies the shamanic state of consciousness shifts the brain state to the upper limit of the critical zone. With heightened criticality, the brains of shamanic practitioners may be more susceptible to internal and external perturbations, which produce more diverse spatiotemporal brain activity patterns and consequently allow for a "richer" or more "diverse" conscious experience. This is supported by the correlations between increased PCF in the low beta band and the OAV domains of complex imagery and elementary visual alterations. Furthermore, while PCF increases were greatest during shamanic drumming, PCF was also increased during classical music and eye closure in shamanic practitioners compared to the control group, suggesting that shamanic practice may induce enduring changes in criticality, shifting the brain to reside in the upper bounds of the critical zone long-term (i.e., over months or years). Future longitudinal studies could expand these findings by examining criticality and other EEG measures at various points within the lifetime of shamanic practitioners.

\section{METHODOLOGIC STRENGTHS AND WEAKNESSES}

This study has many strengths. In addition to validating the spectral changes noted in the only previous EEG study of shamanic trance, we characterized the shamanic state of consciousness using a variety of computational methods used previously to assess other altered states of consciousness. Furthermore, we investigated the relationship between such measures and changes in consciousness reported by shamanic practitioners. This allowed for the most comprehensive characterization of the shamanic state of consciousness to date while using the largest cohort of shamanic practitioners reported in the literature. Finally, this study allowed for the investigation of changes in phenomenal content in the absence of pharmacologic manipulation. Given that psychedelic agents indiscriminately act on brain regions based solely on their receptor profile, the use of these substances may produce confounding results by altering attributes of cortical functioning unrelated to the altered state of consciousness being probed. Thus, the ability to study altered states while free of pharmacologic agents may provide a more direct approach to elucidating the neural correlates of consciousness.

This study has some limitations. First, we did not collect electromyography data to control for movement, meaning it remains possible that our findings within the gamma band are contaminated by muscle artifact. Additionally, our sample size was small relative to many neuroimaging studies. This led to the lack of adjustment for multiple comparisons in our post hoc analyses due to their exploratory nature. Yet, it is important to note that trend relationships ( $p=0.052-0.07$ ) would remain for a majority of EEG measures (connectivity, signal diversity) and almost all PCF results, excluding in the 
high beta band during music $(p=0.065)$, would remain significant $(p<0.05)$ after correction (Benjamini-Hochberg procedure). It is unknown whether the relationships between EEG measures and OAV domain scores would have survived adjustment for multiple comparisons, meaning these exploratory findings should be interpreted with a degree of caution. Future studies should aim to include a larger cohort of shamanic practitioners that is powered to formally investigate this relationship.

Our cohort of shamanic practitioners had diverse backgrounds of shamanic practice and rituals, which introduced potential variability in our study population while also allowing for greater generalizability of findings and ease of recruitment. A summary of the shamanic traditions utilized by each practitioner during normal, non-study shamanic trance sessions is detailed in Supplementary Table 3.

Another limitation of this study is that our control participants were asked to rest quietly during the shamanic drumming period while our shamanic practitioners were asked to enter a shamanic trance. This means shamanic practitioners were cognitively engaged during this time while controls were passively listening, which could have led to a difference in EEG changes. However, unlike previous studies of the shamanic state of consciousness, we collected data during classical music as a contrast condition to identify brain activity specific to shamanic trance rather than due to cognitive engagement. Furthermore, a recent study investigating the effects of rhythmic drumming on sparse EEG in healthy volunteers found that the suggestion to enter a trance state during rhythmic drumming elicited increased delta and decreased alpha activity, which the authors attributed to increased relaxation or drowsiness rather than effects of a trance state (Konopacki and Madison, 2018). Thus, our instruction for healthy volunteers to only listen during shamanic drumming was likely still a comparable control condition.

It is important to note that there were methodological differences between the current study and that of Studerus et al. that may potentially complicate the comparison of OAV domain scores from shamanic practitioners and healthy volunteers under the influence of psychedelics. First, the data used by Studerus et al. (2010) was aggregated from 43 studies which varied by methodology and dose. Specifically, doses for each of the three drugs varied as follows: psilocybin $115-350 \mu \mathrm{g} / \mathrm{kg}$ orally (low to high dose); ketamine $6-12 \mu \mathrm{g} \mathrm{kg}^{-1} \mathrm{~min}^{-1}$ intravenously (medium to high dose); MDMA varied from $1.5-1.7 \mathrm{mg} / \mathrm{kg}$ orally (medium dose; Studerus et al., 2010). Thus, the OAV scores from Studerus et al. represent the average dose across 43 studies rather than OAV scores of individuals under a traditional low, medium, or high dose. However, given that we are unsure of the strength or "dose" of the shamanic trance each practitioner endured, this comparison is somewhat preferable. Some shamanic practitioners in our study may have entered a "high dose" trance during drumming (similar to a high dose of a psychedelic) whereas others may have had an experience equivalent to a "low dose". Additionally, while shamanic practitioners kept their eyes closed during shamanic drumming, methodology varied across the studies included by
Studerus et al. (2010). Recent literature examining the impact of external stimulation during the psychedelic state found that the greatest changes in brain activity (i.e., brain entropy) occurred during eye closure (Mediano et al., 2020). Thus, the heightened OAV scores in shamanic practitioners compared to those of individuals under the influence of psychedelics may be due to the inclusion of less intense experiences due to eye-opening.

Finally, there was a lack of blinding between experimental conditions or groups, which could have led to exaggerated OAV scores in shamanic practitioners or decreased OAV scores in the control group due to demand characteristics. However, we attempted to reduce expectancies by randomizing the order of experimental conditions.

\section{CONCLUSION}

We found that shamanic practitioners entered an altered state of consciousness compared to control participants during drumming, with the magnitude of these changes in consciousness comparable to or greater than reports during the psychedelic state. Furthermore, shamanic practitioners evinced changes in a variety of EEG measures during shamanic drumming that correlated with measures of their altered states of consciousness. These findings suggest that shamanic trance and psychedelic drug-induced altered states of consciousness are "distinct states with shared traits," meaning that despite various overlapping phenomenal components, they are characterized by different changes in brain activity. This suggests the involvement of endogenous mechanisms for non-pharmacologically-induced altered states of consciousness that are distinct from those co-opted by psychedelic drugs.

\section{DATA AVAILABILITY STATEMENT}

The raw data supporting the conclusions of this article will be made available by the authors, without undue reservation.

\section{ETHICS STATEMENT}

The studies involving human participants were reviewed and approved by University of Michigan Institutional Review Board (HUM00089064). The patients/participants provided their written informed consent to participate in this study.

\section{AUTHOR CONTRIBUTIONS}

$\mathrm{EH}$ and HK: formal analysis, writing-original draft, writing-review and editing. UL: methodology, writing-review and editing. TB-B and AC: data curation, writing-review and editing. AN: data curation. SB-M: conceptualization, methodology, writing-review and editing. GM: conceptualization, funding acquisition, resources, writing-review and editing. RH: conceptualization, supervision, methodology, resources, writing-review and editing. 


\section{FUNDING}

This work was funded by the Center for Consciousness Science, Department of Anesthesiology, University of Michigan Medical School.

\section{ACKNOWLEDGMENTS}

We would like to thank Dr. Erich Studerus for his agreement to and helpful suggestions for comparing our data from shamanic practitioners to healthy volunteers under the influence

\section{REFERENCES}

Abdullah, A. A., and Omar, Z. (2011). The effect of temporal EEG signals while listening to quran recitation. Int. J. Adv. Sci. Eng. Inform. Technol. 1, 372-375. doi: $10.18517 /$ ijaseit.1.4.77

Al-Galal, S. A., and Alshaikhli, I. F. T. (2017). Analyzing brainwaves while listening to quranic recitation compared with listening to music based on EEG signals. Int. J. Percept. Cogn. Comput. 3:1. doi: 10.31436/ijpcc. $\mathrm{v} 3 \mathrm{i} 1.43$

Atasoy, S., Roseman, L., Kaelen, M., Kringelbach, M. L., Deco, G., and CarhartHarris, R. L. (2017). Connectome-harmonic decomposition of human brain activity reveals dynamical repertoire re-organization under LSD. Sci. Rep. 7:17661. doi: 10.1038/s41598-017-17546-0

Bak, P., Tang, C., and Wiesenfeld, K. (1988). Self-organized criticality. Phys. Rev. A Gen. Phys. 38, 364-374. doi: 10.1103/physreva.38.364

Barcelona, J., Fahlman, M., Churakova, Y., Canjels, R., Mallare, J., and van den Heuvel, M. I. (2020). Frontal $\alpha$ asymmetry during prayerful and resting states: an EEG study in Catholic sisters. Int. J. Psychophysiol. 155, 9-15. doi: 10.1016/j. ijpsycho.2020.04.019

Bastos, M. A. V. Jr., Bastos, P. R. H. O., Osório, I. H. S., Muass, K. A. R. C., Iandoli, D. Jr., Lucchetti, G., et al. (2016). Frontal electroencephalographic (EEG) activity and mediumship: a comparative study between spiritist mediums and controls. Arch. Clin. Psychiatry 43, 20-26. doi: 10.1590/010160830000000076

Beauregard, M., and Paquette, V. (2008). EEG activity in Carmelite nuns during a mystical experience. Neurosci. Lett. 444, 1-4. doi: 10.1016/j.neulet.2008. 08.028

Beethoven, L. (1801/2013). Sonata No.5 in F Major for Violin and Piano, Op. 24, "Spring": I. Allegro. [Song recorded by N. Chastain and F. Rieger] On My First Beethoven Album. X5 Music Group.

Berkovich-Ohana, A., Glicksohn, J., and Goldstein, A. (2012). Mindfulnessinduced changes in $\gamma$ band activity-implications for the default mode network, self-reference and attention. Clin. Neurophysiol. 123, 700-710. doi: 10.1016/j.clinph.2011.07.048

Bodmer, I., Dittrich, A., and Lamparter, D. (1994). Aussergewöhnliche Bewusstseinszustände-Ihre Gemeinsame Struktur und Messung [Altered States of Consciousness-Their Common Structure and Assessment]. In: Welten des Bewusstseins. Bd. 3 Experimentelle Psychologie, Neurobiologie und Chemie. Berlin: Germany.

Braboszcz, C., Cahn, B. R., Levy, J., Fernandez, M., and Delorme, A. (2017). Increased $\gamma$ brainwave amplitude compared to control in three different meditation traditions. PLoS One 12:e0170647. doi: 10.1371/journal.pone. 0170647

Cahn, B. R., Delorme, A., and Polich, J. (2010). Occipital $\gamma$ activation during Vipassana meditation. Cogn. Process. 11, 39-56. doi: 10.1007/s10339-009 $-0352-1$

Carhart-Harris, R. L., Erritzoe, D., Williams, T., Stone, J. M., Reed, L. J., Colasanti, A., et al. (2012). Neural correlates of the psychedelic state as determined by fMRI studies with psilocybin. Proc. Natl. Acad. Sci. U S A 109, 2138-2143. doi: 10.1073/pnas.1119598109

Carhart-Harris, R. L., Leech, R., Hellyer, P. J., Shanahan, M., Feilding, A., Tagliazucchi, E., et al. (2014). The entropic brain: a theory of conscious of psychedelics. Additionally, we would like to thank Kate Durda and Stephanie Tighe, who offered invaluable insight as shamanic practitioners when designing our study. This manuscript has been released as a pre-print at bioRxiv (Huels et al., 2020).

\section{SUPPLEMENTARY MATERIAL}

The Supplementary Material for this article can be found online at: https://www.frontiersin.org/articles/10.3389/fnhum. 2021.610466/full\#supplementary-material.

states informed by neuroimaging research with psychedelic drugs. Front. Hum. Neurosci. 8:20. doi: 10.3389/fnhum.2014.00020

Carhart-Harris, R. L., Muthukumaraswamy, S., Roseman, L., Kaelen, M., Droog, W., Murphy, K., et al. (2016). Neural correlates of the LSD experience revealed by multimodal neuroimaging. Proc. Natl. Acad. Sci. U S A 113, 4853-4858. doi: 10.1073/pnas.1518377113

Delorme, A., and Makeig, S. (2004). EEGLAB: an open source toolbox for analysis of single-trial EEG dynamics. J. Neurosci. Methods 134, 9-21. doi: 10.1016/j. jneumeth.2003.10.009

Don, N. S., McDonough, B. E., Moura, G., Warren, C. A., Kawanishi, K., Tomita, H., et al. (1998). Effects of Ayahuasca on the human EEG. Phytomedicine 5, 87-96. doi: 10.1016/S0944-7113(98)80003-2

Doufesh, H., Faisal, T., Lim, K.-S., and Ibrahim, F. (2012). EEG spectral analysis on Muslim prayers. Appl. Psychophysiol. Biofeedback 37, 11-18. doi: 10.1007/s10484-011-9170-1

Doufesh, H., Ibrahim, F., Ismail, N. A., and Wan Ahmad, W. A. (2014). Effect of Muslim prayer (Salat) on $\alpha$ electroencephalography and its relationship with autonomic nervous system activity. J. Altern. Complement. Med. 20, 558-562. doi: 10.1089/acm.2013.0426

Ewerdwalbesloh, J. A., Palva, S., Rösler, F., and Khader, P. H. (2016). Neural correlates of maintaining generated images in visual working memory. Hum. Brain Mapp. 37, 4349-4362. doi: 10.1002/hbm. 23313

Fell, J., Axmacher, N., and Haupt, S. (2010). From $\alpha$ to $\gamma$ : electrophysiological correlates of meditation-related states of consciousness. Med. Hypotheses 75, 218-224. doi: 10.1016/j.mehy.2010.02.025

Fiorica-Howells, E., Hen, R., Gingrich, J., Li, Z., and Gershon, M. D. (2002). 5 HT2A receptors: location and functional analysis in intestines of wild-type and 5-HT2A knockout mice. Am. J. Physiol. Gastrointest. Liver Physiol. 282, G877-G893. doi: 10.1152/ajpgi.00435.2001

Flor-Henry, P., Shapiro, Y., and Sombrun, C. (2017). Brain changes during a shamanic trance: altered modes of consciousness, hemispheric laterality and systemic psychobiology. Cogent Psychol. 4:1313522. doi: 10.1080/23311908. 2017.1313522

Halberstadt, A. L., and Geyer, M. A. (2011). Multiple receptors contribute to the behavioral effects of indoleamine hallucinogens. Neuropharmacology 61, 364-381. doi: 10.1016/j.neuropharm.2011.01.017

Harner, M. (1980). The Way of the Shaman. 3rd Edn. San Francisco: Harper and Row.

Harner, M. (1997). "15 minute solo drumming with call back", in Michael Harner's Shamanic Journey Solo and Double Drumming. Mill Valley, CA: The Foundation for Shamanic Studies, Inc.

Havlík, M. (2017). Missing piece of the puzzle in the science of consciousness: resting state and endogenous correlates of consciousness. Conscious. Cogn. 49, 70-85. doi: 10.1016/j.concog.2017.01.006

Hove, M. J., Stelzer, J., Nierhaus, T., Thiel, S. D., Gundlach, C., Margulies, D. S., et al. (2016). Brain network reconfiguration and perceptual decoupling during an absorptive state of consciousness. Cereb. Cortex 26, 3116-3124. doi: $10.1093 /$ cercor/bhv137

Huels, E. R., Kim, H., Lee, U., Bel-Bahar, T., Colmenero, A., Nelson, A., et al. (2020). Neural correlates of the shamanic state of consciousness. bioRxiv [Preprint]. doi: 10.1101/2020.07.20.212522 
Johnson, M. W., Hendricks, P. S., Barrett, F. S., and Griffiths, R. R. (2019). Classic psychedelics: an integrative review of epidemiology, therapeutics, mystical experience, and brain network function. Pharmacol. Ther. 197, 83-102. doi: 10.1016/j.pharmthera.2018.11.010

Kakumanu, R. J., Nair, A. K., Venugopal, R., Sasidharan, A., Ghosh, P. K., John, J. P., et al. (2018). Dissociating meditation proficiency and experience dependent EEG changes during traditional Vipassana meditation practice. Biol. Psychol. 135, 65-75. doi: 10.1016/j.biopsycho.2018.03.004

Kawai, N., Honda, M., Nishina, E., Yagi, R., and Oohashi, T. (2017). Electroencephalogram characteristics during possession trances in healthy individuals. Neuroreport 28, 949-955. doi: 10.1097/WNR.0000000000000857

Kim, H., and Lee, U. (2019). Criticality as a determinant of integrated information $\Phi$ in human brain networks. Entropy 21:981. doi: 10.3390/e21100981

Kim, M., Kim, S., Mashour, G. A., and Lee, U. (2017). Relationship of topology, multiscale phase synchronization, and state transitions in human brain networks. Front. Comput. Neurosci. 11:55. doi: 10.3389/fncom.2017.00055

Kim, M., Mashour, G. A., Moraes, S.-B., Vanini, G., Tarnal, V., Janke, E., et al. (2016). Functional and topological conditions for explosive synchronization develop in human brain networks with the onset of anesthetic-induced unconsciousness. Front. Comput. Neurosci. 10:1. doi: 10.3389/fncom.2016. 00001

Kitzbichler, M. G., Smith, M. L., Christensen, S. R., and Bullmore, E. (2009). Broadband criticality of human brain network synchronization. PLoS Comput. Biol. 5:e1000314. doi: 10.1371/journal.pcbi.1000314

Kometer, M., Pokorny, T., Seifritz, E., and Volleinweider, F. X. (2015). Psilocybin-induced spiritual experiences and insightfulness are associated with synchronization of neuronal oscillations. Psychopharmacology 232, 3663-3676. doi: $10.1007 / \mathrm{s} 00213-015-4026-7$

Kometer, M., Schmidt, A., Jäncke, L., and Vollenweider, F. X. (2013). Activation of serotonin $2 \mathrm{~A}$ receptors underlies the psilocybin-induced effects on $\alpha$ oscillations, N170 visual-evoked potentials and visual hallucinations. J. Neurosci. 33, 10544-10551. doi: 10.1523/JNEUROSCI.3007-12.2013

Konopacki, M., and Madison, G. (2018). EEG responses to shamanic drumming. Does the suggestion of trance state moderate the strength of frequency components? J. Sleep Sleep Disord. Res. 1, 16-25. doi: 10.14302/issn.2574-4518. jsdr-17-1794

Kraehenmann, R., Pokorny, D., Vollenweider, L., Preller, K. H., Pokorny, T., Seifritz, E., et al. (2017). Dreamlike effects of LSD on waking imagery in humans depend on serotonin 2A receptor activation. Psychopharmacology 234, 2031-2046. doi: 10.1007/s00213-017-4610-0

Krippner, S. (2000). The epistemology and technologies of shamanic states of consciousness. J. Conscious. Stud. 7, 93-118.

Lee, H., Golkowski, D., Jordan, D., Berger, S., Ilg, R., Lee, J., et al. (2019). Relationship of critical dynamics, functional connectivity and states of consciousness in large-scale human brain networks. NeuroImage 188, 228-238. doi: 10.1016/j.neuroimage.2018.12.011

Lee, U., and Mashour, G. A. (2018). The role of network science in the study of anesthetic state transitions. Anesthesiology 129, 1029-1044. doi: 10.1097/ALN. 0000000000002228

Lee, M., Sanders, R. D., Yeom, S.-K., Won, D.-O., Kim, H.-J., Lee, B.-R., et al. (2017). Change in functional networks for transitions between states of consciousness during midazolam-induced sedation. Conf. Proc. IEEE Eng. Med. Biol. Soc. 2017, 958-961. doi: 10.1109/EMBC.2017.8036984

Leemburg, S., and Bassetti, C. L. (2018). Lempel-Ziv complexity of the EEG predicts long-term functional recovery after stroke in rats. bioRxiv [Preprint]. doi: $10.1101 / 248039$

Lempel, A., and Ziv, J. (1976). On the complexity of finite sequences. IEEE Trans. Inform. Theor. 22, 75-81. doi: 10.1109/tit.1976.1055501

Li, D., and Mashour, G. A. (2019). Cortical dynamics during psychedelic and anesthetized states induced by ketamine. NeuroImage 196, 32-40. doi: 10.1016/j.neuroimage.2019.03.076

Li, D., Vlisides, P. E., Kelz, M. B., Avidan, M. S., Mashour, G. A., and ReCCognition Study Group. (2019). Dynamic cortical connectivity during general anesthesia in healthy volunteers. Anesthesiology 130, 870-884. doi: 10.1097/ALN. 0000000000002656

Luft, C. D. B., Zioga, I., Banissy, M. J., and Bhattacharya, J. (2019). Spontaneous visual imagery during meditation for creating visual art: an EEG and brain stimulation case study. Front Psychol 10:210. doi: 10.3389/fpsyg.2019.00210
Lutz, A., Greischar, L. L., Rawlings, N. B., Ricard, M., and Davidson, R. J. (2004). Long-term meditators self-induce high-amplitude $\gamma$ synchrony during mental practice. Proc. Natl. Acad. Sci. U S A 101, 16369-16373. doi: 10.1073/pnas. 0407401101

Martínez Vivot, R., Pallavicini, C., Zamberlan, F., Vigo, D., and Tagliazucchi, E. (2020). Meditation increases the entropy of brain oscillatory activity. Neuroscience 431, 40-51. doi: 10.1016/j.neuroscience.2020.01.033

Martinovic, J., and Busch, N. A. (2011). High frequency oscillations as a correlate of visual perception. Int. J. Psychophysiol. 79, 32-38. doi: 10.1016/j.ijpsycho. 2010.07.004

Mediano, P. A. M., Rosas, F. E., Timmermann, C., Roseman, L., Nutt, D. J., Feilding, A., et al. (2020). Effects of external stimulation on psychedelic state neurodynamics. bioRxiv [Preprint]. doi: 10.1101/2020.11.01. 356071

Moretti, P., and Muñoz, M. A. (2013). Griffiths phases and the stretching of criticality in brain networks. Nat. Commun. 4:2521. doi: 10.1038/ncomms3521

Mozart, W. A. (1788/2009). Sonata for Piano No. 15 in F Major, K. 533: I. Allegro. [Song recorded by C. Piazzini]. On Mozart: The Complete Piano Sonatas. Cascade.

Muñoz, M. A. (2018). Colloquium: criticality and dynamical scaling in living systems. Rev. Mod. Phys. 90:031001. doi: 10.1103/revmodphys.90.031001

Muthukumaraswamy, S. D., Carhart-Harris, R. L., Moran, R. J., Brookes, M. J., Williams, T. M., Errtizoe, D., et al. (2013). Broadband cortical desynchronization underlies the human psychedelic state. J. Neurosci. 33, 15171-15183. doi: 10.1523/JNEUROSCI.2063-13.2013

O’Mahony, S. M., Clarke, G., Borre, Y. E., Dinan, T. G., and Cryan, J. F. (2015). Serotonin, tryptophan metabolism and the brain-gut-microbiome axis. Behav. Brain Res. 277, 32-48. doi: 10.1016/j.bbr.2014.07.027

Oohashi, T., Kawai, N., Honda, M., Nakamura, S., Morimoto, M., Nishina, E., et al. (2002). Electroencephalographic measurement of possession trance in the field. Clin. Neurophysiol. 113, 435-445. doi: 10.1016/s1388-2457(02) 00002-0

Pal, D., Li, D., Dean, J. G., Brito, M. A., Liu, T., Fryzel, A. M., et al. (2020). Level of consciousness is dissociable from electroencephalographic measures of cortical connectivity, slow oscillations and complexity. J. Neurosci. 40, 605-618. doi: 10.1523/JNEUROSCI.1910-19.2019

Palhano-Fontes, F., Andrade, K. C., Tofoli, L. F., Santos, A. C., Crippa, J. A. S., Hallak, J. E. C., et al. (2015). The psychedelic state induced by ayahuasca modulates the activity and connectivity of the default mode network. PLoS One 10:e0118143. doi: 10.1371/journal.pone.0118143

Pallavicini, C., Vilas, M. G., Villarreal, M., Zamberlan, F., Muthukumaraswamy, S., Nutt, D., et al. (2019). Spectral signatures of serotonergic psychedelics and glutamatergic dissociatives. NeuroImage 200, 281-291. doi: 10.1016/j. neuroimage.2019.06.053

Preller, K. H., Burt, J. B., Ji, J. L., Schleifer, C. H., Adkinson, B. D., Stämpfli, P., et al. (2018). Changes in global and thalamic brain connectivity in LSD-induced altered states of consciousness are attributable to the 5-HT2A receptor. Elife 7:e35082. doi: 10.7554/eLife.35082

Riba, J., Anderer, P., Jané, F., Saletu, B., and Barbanoj, M. J. (2004). Effects of the South American psychoactive beverage ayahuasca on regional brain electrical activity in humans: a functional neuroimaging study using low-resolution electromagnetic tomography. Neuropsychobiology 50, 89-101. doi: 10.1159/000077946

Riba, J., Anderer, P., Morte, A., Urbano, G., Jané, F., Saletu, B., et al. (2002). Topographic pharmaco-EEG mapping of the effects of the South American psychoactive beverage ayahuasca in healthy volunteers. Br. J. Clin. Pharmacol. 53, 613-628. doi: 10.1046/j.1365-2125.2002.01609.x

Schartner, M. M., Carhart-Harris, R. L., Barrett, A. B., Seth, A. K., and Muthukumaraswamy, S. D. (2017). Increased spontaneous MEG signal diversity for psychoactive doses of ketamine, LSD and psilocybin. Sci. Rep. 7:46421. doi: 10.1038/srep46421

Schenberg, E. E., Alexandre, J. F. M., Filev, R., Cravo, A. M., Sato, J. R., Muthukumaraswamy, S. D., et al. (2015). Acute biphasic effects of ayahuasca. PLoS One 10:e0137202. doi: 10.1371/journal.pone.0137202

Stam, C. J., Nolte, G., and Daffertshofer, A. (2007). Phase lag index: assessment of functional connectivity from multi channel EEG and MEG with diminished bias from common sources. Hum. Brain Mapp. 28, 1178-1193. doi: $10.1002 / \mathrm{hbm} .20346$ 
Studerus, E., Gamma, A., and Vollenweider, F. X. (2010). Psychometric evaluation of the altered states of consciousness rating scale (OAV). PLoS One 5:e12412. doi: 10.1371/journal.pone.0012412

Timmermann, C., Roseman, L., Schartner, M., Milliere, R., Williams, L. T. J., Erritzoe, D., et al. (2019). Neural correlates of the DMT experience assessed with multivariate EEG. Sci. Rep. 9:16324. doi: 10.1038/s41598-019-51974-4

Tributsch, H. (2018). Shamanic trance journey with animal spirits: ancient "scientific" strategy dealing with inverted otherworld. Adv. Anthropol. 8, 91-126. doi: 10.4236/aa.2018.83006

Vaghefi, M., Nasrabadi, A. M., Golpayegani, S. M. R. H., Mohammadi, M.-R., and Gharibzadeh, S. (2015). Spirituality and brain waves. J. Med. Eng. Technol. 39, 153-158. doi: 10.3109/03091902.2014.1001528

Vaghefi, M., Nasrabadi, A. M., Hashemi Golpayegani, S. M. R., Mohammadi, M. R., and Gharibzadeh, S. (2019). Nonlinear analysis of electroencephalogram signals while listening to the holy quran. J. Med. Signals Sens. 9, 100-110. doi: 10.4103/jmss.JMSS_37_18

Valle, M., Maqueda, A. E., Rabella, M., Rodríguez-Pujadas, A., Antonijoan, R. M., Romero, S., et al. (2016). Inhibition of $\alpha$ oscillations through serotonin-2A receptor activation underlies the visual effects of ayahuasca in humans. Eur. Neuropsychopharmacol. 26, 1161-1175. doi: 10.1016/j.euroneuro. 2016.03.012

Vinck, M., Oostenveld, R., van Wingerden, M., Battaglia, F., and Pennartz, C. M. A. (2011). An improved index of phase-synchronization for electrophysiological data in the presence of volume-conduction, noise and sample-size bias. NeuroImage 55, 1548-1565. doi: 10.1016/j.neuroimage.2011.01.055

Winkelman, M. J. (2013). Shamanism and psychedelics: a biogenetic structuralist paradigm of ecopsychology. Eur. J. Ecopsychol. 4, 90-115.

Yoon, S., Sorbaro Sindaci, M., Goltsev, A. V., and Mendes, J. F. F. (2015). Critical behavior of the relaxation rate, the susceptibility and a pair correlation function in the Kuramoto model on scale-free networks. Phys. Rev. E Stat. Nonlin. Soft Matter Phys. 91:032814. doi: 10.1103/PhysRevE.91.032814

Zhang, X. S., Roy, R. J., and Jensen, E. W. (2001). EEG complexity as a measure of depth of anesthesia for patients. IEEE Trans. Biomed. Eng. 48, 1424-1433. doi: 10.1109/10.966601

Conflict of Interest: The authors declare that the research was conducted in the absence of any commercial or financial relationships that could be construed as a potential conflict of interest.

Copyright (c) 2021 Huels, Kim, Lee, Bel-Bahar, Colmenero, Nelson, Blain-Moraes, Mashour and Harris. This is an open-access article distributed under the terms of the Creative Commons Attribution License (CC BY). The use, distribution or reproduction in other forums is permitted, provided the original author(s) and the copyright owner(s) are credited and that the original publication in this journal is cited, in accordance with accepted academic practice. No use, distribution or reproduction is permitted which does not comply with these terms. 